\title{
Sars-Cov-2 Un Análisis Bibliométrico en La Actualidad
}

\section{An Updated Bibliometric Analysis on Sars-Cov-2}

\section{Manuel Llerena ${ }^{1 *} \&$ Manuel Arévalo ${ }^{2}$}

\author{
${ }^{1}$ Universidad Católica de Santiago de Guayaquil. Facultad de Ciencias Económicas y \\ Administrativas. Av. Carlos Julio Arosemena, km $1^{1 / 2}$, Guayaquil (Ecuador). \\ ${ }^{2}$ Tecnológico Universitario Espíritu Santo. Carrera de Sistemas. Av. Juan Tanga Marengo y \\ Av. Las Aguas, Guayaquil (Ecuador).
}

Recibido 3 agosto 2021; revisado 26 agosto 2021; aceptado noviembre 2021; disponible en línea 30 de diciembre 2021.

\section{Resumen}

En Wuhan se detectó los primeros y únicos casos por un nuevo coronavirus, denominado SARSCoV-2 que es una enfermedad respiratoria que presenta en sus infectados síntomas leves y en otros tipos de pacientes puede llegar a desarrollar situaciones mortíferas. El objetivo primordial del presente estudio fue analizar cómo ha evolucionado la producción científica del covid-19 a través de un estudio bibliométrico. Dentro de la implementación de materiales y métodos se efectuó una búsqueda bibliográfica a través de tres bases de datos de alto prestigio como Web of science, Scopus y PubMed donde se identificaron varios estratos estructurales relacionados al país de procedencia, autores más productivos y palabras claves. Teniendo en consideración que los resultados mostraron el análisis de 98.458 artículos científicos de cuartil 1 - 2 el cual identificó a Chinese Academy of Science como la organización que forma parte de la élite en investigaciones de la covid-19, no obstante, se identificó que la base de datos que mayor aportó en la presente investigación fue Web of Science con un total de 46.418 artículos arbitrados en el año 2021 y que la palabra clave más buscada a en todo el mundo fue la covid-19. Finalmente, el estudio evidenció la participación de cada una de las unidades de educación superior, países del primer mundo y expertos sobre el SARS-CoV-2 trabajando en conjunto para obtener la mayor cantidad de respuestas acerca de este virus que paralizó al mundo.

Palabras clave: Bibliometría, coronavirus, covid-19, sars-cov-2, 2019-ncov2.

\begin{abstract}
In Wuhan, the first and only cases of a new coronavirus were detected, called SARS-CoV-2, which is a respiratory disease that presents mild symptoms in its infected and in other types of patients it can develop deadly situations. The main objective of this study was to analyze how the scientific production of covid-19 has evolved through a bibliometric study. Within the implementation of materials and methods, a bibliographic search was carried out through three highly prestigious databases such as Web of science, Scopus and PubMed, where several structural strata related to the country of origin, the most productive authors and keywords were identified. Taking into consideration that the results showed the analysis of 98,458 scientific articles from quartile $1-2$ which identified the Chinese Academy of Science as the organization that is part of the elite in covid-19 research, however it was identified that the base of data that contributed the most in this research was Web Of Science with a total of 46,418 articles refereed in the year 2021 and that the most searched keyword worldwide was covid-19. Finally, the study evidenced the participation of each of the higher education units, first world countries and experts on SARS-CoV-2 working together to obtain the highest number of answers about this virus that paralyzed the world.

Key words: Bibliometrics, coronavirus, covid-19, sars-cov-2, 2019-ncov2.
\end{abstract}

\section{Introducción}

El domingo 1 de diciembre del 2019 a las 15:34:43 pm en Wuhan, se detectaron los primeros y únicos casos por un nuevo coronavirus, denominado SARSCoV-2, que fue notificado por el instituto de Virología de Wuhan, uno de los prestigiosos centros científicos en China (O'Brien et al., 2020). Autores como Vásquez et al. (2021) manifestaron que la covid-19 es una enfermedad respiratoria que presenta en sus infectados síntomas leves como: a) tos seca, b) escalofríos, c) fiebre, d) insomnio, e) dolor de

* Correspondencia del autor:

E-mail: manuel.llerena@cu.ucsg.edu.ec

2017 Facultad de Ciencias Naturales de la Universidad de Guayaquil. Este obra está bajo una licencia de Creative Commons Reconocimiento-NoComercial-SinObraDerivada 4.0 Internacional 
Rev. Cient. Cien. Nat. Ambien. 15(2):289-298

Diciembre 2021
ISSN: $2773-7772$
Llerena • Sars-Cov-2 Un Análisis Bibliométrico en La Actualidad garganta, f) sudoración excesiva, etc. Mientras que Cheng et al. (2020) recalcaron que en otros tipos de pacientes puede llegar a desarrollar situaciones mortíferas, como: a) dificultad aguda respiratoria, b) shock séptico, c) neumonía, y d) edemas pulmonares. Por otra parte, Wang y Liao (2020) señalaron que existen diferentes tipos de virus, la coronavirus gamma y delta pertenecen a especies de aves (gallinas, patos, palomas, etc...) que apenas causan infecciones humanas, mientras que la coronavirus alfa y beta se hallan principalmente en mamíferos (murciélagos, roedores, civetas, etc...) y humanos que por ende se conoce que causan enfermedades humanas; la mayoría de infecciones por coronavirus en humanos específicamente en virus denominados HCoV-229E, HCoV-OC43, HCoV-NL63, HCoV-HKU1 presentan síntomas leves como el resfriado común y la diarrea en los seres humanos. Sin embargo, Gregorio et al. (2020) recalcaron que en varios estudios la genética del virus ha variado en relación a las características genómicas de SARS-CoV-2 usando secuencia de muestras liquidad de lavado broncoalveolar y aislamientos de diferentes cultivos de pacientes contagiados con el virus, se debe tener en consideración que el SARS-CoV-2 muestra un nuevo virus dentro del subgrupo de sarbecovirus el cual se clasifica en tres segmentos: a) SARS-CoV-2 de Wuhan junto con las dos cepas similares al SARS derivadas de murciélagos, bat-SL-CoVZC45 y bat-SL-CoVZXC21 de Zhoushan, Zhejiang, China; b) cepas de SARS-CoV de humanos y algunas genéticamente coronavirus similares al SARS de murciélagos detectados en sur oeste de China; y c) dos cepas relacionadas con el SARS-CoV de Rhinolophus de Bulgaria y Kenia (Shuai et al., 2020; Wu et al., 2020; Zhou et al., 2020). Giwan et al. (2020), evidenciaron detalladamente la detección rápida del virus causante del covid-19 (SARS-CoV-2) en humanos nasofaríngeos a través de muestras de hisopo usando el transistor de efecto de campo (fig. 1).

Nota. El primer paso es tomar una muestra hisopada del paciente, el segundo paso es asegurar la evidencia del hisopado en un tubo de ensayo, el tercer paso es colocar la muestra en el grafeno para que el anticuerpo de pico del SARS-CoV-2 se conjugue en la hoja de grafeno a través de 1 hidroxisuccinimida de ácido pireno butírico que es una molécula de interfaz como conector de sonda lo cual nos permite identificar si el paciente objeto de estudio muestra anomalías en su prueba y concluir con el análisis respectivo. Adaptado de Rapid Detection of COVID-19 Causative Virus (SARS-CoV-2) in Human Nasopharyngeal Swab Specimens Using Field-Effect Transistor- Based Biosensor (p.2), (Giwan et al., 2020).

Cabe recalcar que la Organización Mundial de la Salud (OMS, 2020) compartió la cronología declarada el 27 de abril del 2020 y su última versión actualizada el 29 de junio de 2020 con las siguientes connotaciones:

- El 31 de diciembre del 2019, la Comisión Municipal de Sud de Wuhan notifica un conglomerado de casos de neumonía causados por un nuevo coronavirus.

- El 1 de enero del 2020 la OMS establece un equipo de apoyo de 3 frentes y pone así a la entidad en un estado de emergencia.

- El 4 de enero del 2020 la OMS a través de sus canales oficiales anuncia la existencia de una gran afluencia de casos de neumonía sin fallecimientos en la ciudad de Wuhan.

- El 5 de enero de 2020 la OMS publica su primer arte que contiene una evaluación del riesgo y una serie de recomendaciones para Wuhan.

- El 10 de enero del 2020 la OMS publica un conjunto de orientaciones técnicas con recomendaciones para todos los países tomando como base la experiencia con el SARS y el MEDR.

- El 12 de enero del 2020 China hace pública la secuencia genética del virus causante de la covid-19.

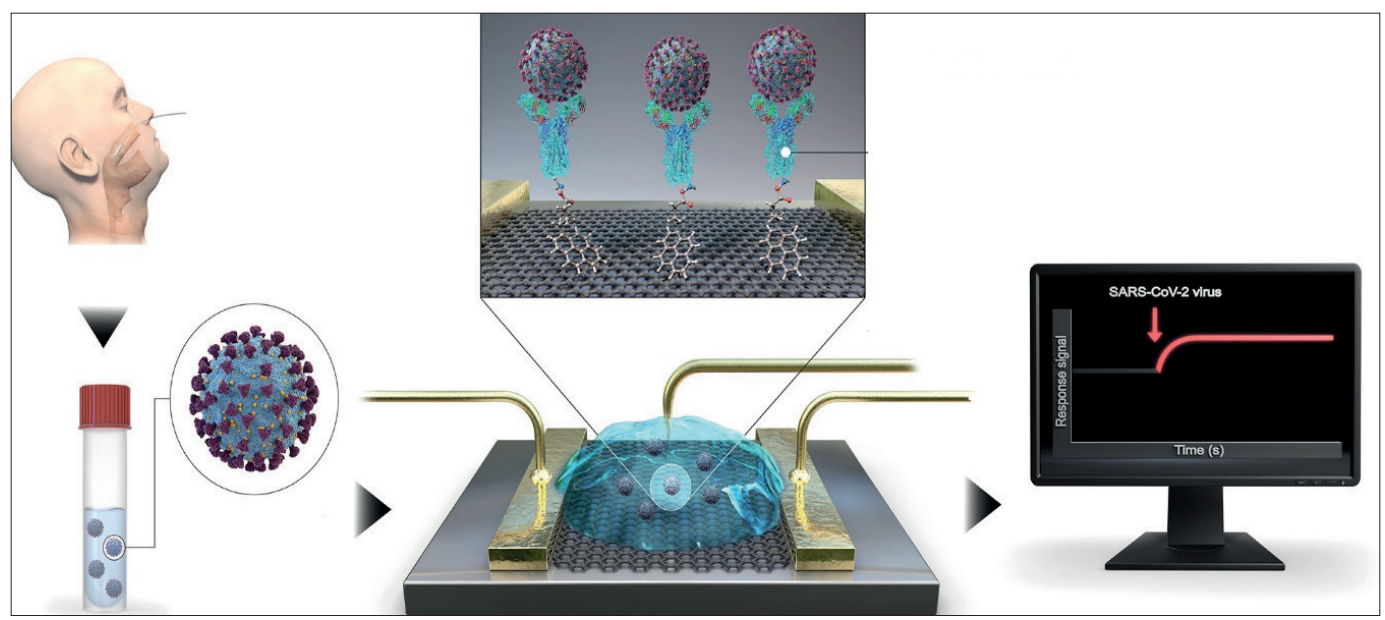

Figura 1. Detección instantánea del virus causante del covid-19. 
- El 13 de enero del 2020 se confirma oficialmente un caso de covid-19 en Tailandia.

- El 14 de enero del 2020 la responsable técnica de la OMS manifiesta que se ha producido una transmisión limitada del coronavirus entre seres humanos.

- Del 20 al 21 de enero del 2020 expertos de la OMS en China efectúan una breve visita sobre el terreno de Wuhan.

- El 22 de enero del 2020 la OMS declara que existe una transmisión entre seres humanos en Wuhan y es necesario más investigaciones para tener la plena seguridad de las aseveraciones.

- Del 22 al 23 de enero del 2020 el Director General convoca a un Comité de Emergencias según lo previsto en el Reglamento Sanitario Internacional para evaluar si el brote constituye una emergencia de salud pública de importancia internacional.

- El 28 de enero del 2020 un grupo de altos funcionarios de la OMS viajan a Beijing para reunirse con los dirigentes de China para conocer mejor el panorama del país y ofrecer asistencia técnica necesaria e intercambiar información y experiencias.

- El 30 de enero del 2020 el Director General convoca de nuevo el Comité de Emergencias, antes de los 10 días de plazo y tan solo dos días después de notificarse los primeros casos de transmisión limitada del coronavirus entre seres humanos

- El 3 de febrero la OMS presenta el Plan Estratégico de Preparación y Respuesta de la comunidad internacional para ayudar a los estados con sistemas de salud más frágiles a protegerse.

- Del 11 al 12 de febrero del 2020 la OMS cita a un foro de investigación e innovación sobre la COVID-19, al que asisten más de 400 expertos y entidades de financiación de todo el mundo.

- Del 16 al 24 de febrero el equipo de la OMS junto a China reúnen expertos de Alemania, Canadá, Estados Unidos, Japón, Nigeria, etc..., los cuales, permanecen un tiempo en Beijing y también viajan a Wuhan para conocer el estado del virus.

- El 11 de marzo del 2020 la OMS específica en su evaluación que la COVID-19 puede caracterizarse como una pandemia, ya que preocupada por los alarmantes niveles de propagación de la enfermedad y por su gravedad.

- El 13 de marzo del 2020 se determina el Fondo de Respuesta Solidaria contra la COVID-19 para recibir donaciones de particulares, empresas e instituciones.

- Finalmente, el 18 de marzo del 2020 la OMS y sus asociados ponen en marcha el ensayo «Solidaridad», que tiene por objeto generar datos sólidos de todo el mundo para encontrar los tratamientos más eficaces contra el virus.

Autores como Pascual y Torrell (2020) manifestaron que se debe agregar que hasta el día de hoy en el mundo existen 250.784.268 casos confirmados, 5.062.943 personas fallecidas y 7.309.958.661 individuos vacunados con las diferentes dosis, la tasa de localización de casos varía día tras día y puede ser visualizada de manera gratuita y desde cualquier dispositivo inteligente en tiempo real ingresando en el sitio web descrito a continuación: https:// gisanddata.maps.arcgis.com/apps/dashboards/ bda7594740fd40299423467b48e9ecf6 donde se podrá observar un sinnúmero de herramientas interactivas que le brindarán la mayor información posible acerca del covid-19 en su región (Bwire \& Paulo, 2020; Lai et al., 2020; Lawani, 1981; Lu et al., 2020; Phat, 2020).

\section{Materiales y métodos}

Para la búsqueda de los documentos se utilizaron las bases de datos como a) Web of science, b) Scopus y c) PubMed; este proceso se elaboró el 2 de agosto del 2021 mediante la siguiente terminología algorítmica [COVID-19-2019-nCoV] o [SARS-CoV-2], con la opción de búsqueda avanzada especificando artículos de cuartil 1 - 2. Cabe recalcar que Llerena et al. (2021) manifestaron la necesidad de normalizar las variables de autores más productivos, filiación institucional entre otras, lo cual es fundamental para la ejecución de los análisis bibliométricos. En la búsqueda ante las 3 bases de datos mencionadas se recuperó un total de 123.541 documentos los cuales se sometieron a un proceso de normalización de metadatos para eliminar documentos réplicas, la muestra idónea para el estudio bibliométrico quedo compuesta por 98.458 documentos (fig. 2) (Chahrour et al., 2020; Dumas et

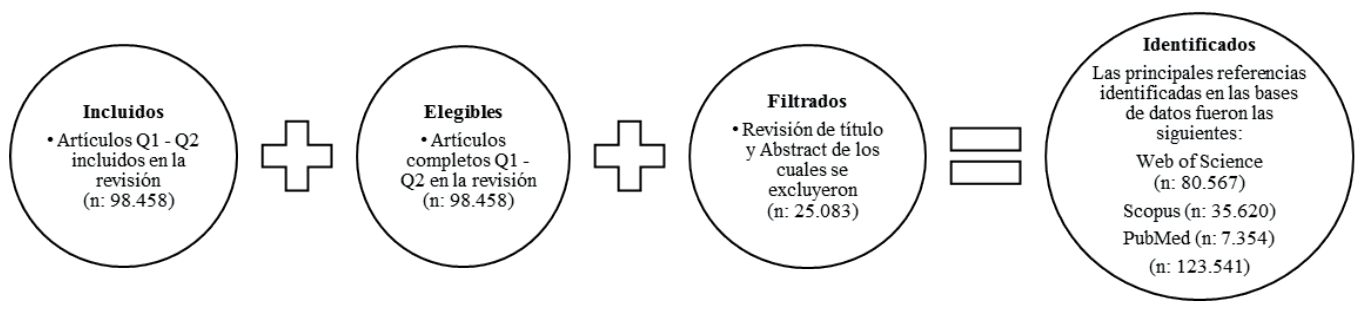

Figura 2. Esquema metodológico de búsqueda, recuperación y selección de la información sobre covid-19. 
Rev. Cient. Cien. Nat. Ambien. 15(2):289-298

Diciembre 2021 al., 2020; Matias-Guiu, 2020; Van \& Waltman, 2010; Zhe et al., 2020).

Nota. Combinación de búsqueda para incluir, elegir, filtrar e identificar los artículos de mayor relevancia para el estudio bibliométrico.

\section{Resultados}

Los resultados están estructurados por la siguiente segmentación: a) autores más productivos, b) filiación institucional, c) año de publicación, d) país de procedencia, e) idioma de publicación, f) áreas de investigaciones, g) editores científicos y h) palabras claves mostrando un top 10 en cada elemento de análisis (Jianju et al., 2020; Kanne, 2020; Lu H, 2020).

\section{Autores más productivos}

La tabla 1 muestra la lista top 10 de los principales autores de covid-19 a nivel internacional, los documentos publicados sobre el tema de estudio las principales citaciones por otros investigadores y el enlace de fuerza entre colaboradores de los distintos países en áreas específicas. Lo cual también se puede apreciar en la figura 3 con un total de 9 clusters.

\section{Filiación institucional}

Por otra parte, la tabla 2 presenta las principales instituciones de educación superior dedicada a la investigación del presente estudio, donde se evidencia el número de documentos publicados, que universidades han usado esas investigaciones y la fuerza de enlace entre instituciones, es necesario recalcar que en la figura 4 se evidencia una formación de 25 cluster con las otras instituciones que no forman parte del top 10.

Año de publicación (área de coronavirus).

En la tabla 3 se demuestran los años de publicación que cada base de datos ha aportado en estudios sobre la covid-19; podemos denotar un total de 158.130 investigaciones durante los 5 años que apareció la primera publicación sobre Sars-CoV-2 a nivel mundial.

\section{País de procedencia.}

En la tabla 4 encontramos los 10 principales países en contribuir con investigaciones sobre la covid-19; sin embargo, se refleja una gran afluencia de otros países en corroborar con sus investigaciones dentro de la actividad científica como se aprecia en la figura 5 conformada por 5 clusters.

Idioma de publicación.

En la tabla 5 es notable observar el idioma de presencia de los artículos publicados en las diferentes revistas de alto impacto y su correspondiente figura 6 con otros idiomas en los que se escriben las diferentes áreas relacionadas.

\section{Áreas de investigaciones}

La tabla 6 muestra cuales son las principales áreas fundamentales de investigaciones acerca del SARSCoV-2, mientras que la figura 7 presenta otras áreas que aportan a los estudios en medicina.

\section{Editores científicos}

En la tabla 7 se presenta el top 10 de los principales editores científicos de cuartil 1 y 2 con aproximadamente un h-index de 235 puntos y además en la figura 8 se demuestra que otros editores contribuyen a las publicaciones relacionadas con el coronavirus.

\section{Palabras claves}

En la tabla 8 se presentan el top 10 de las principales palabras claves de los 98.458 documentos examinados en la presente investigación, de igual manera en la Tabla 8. Top 10 de palabras claves sobre la

Tabla 1. Top 10 de autores más productivos en la temática de covid-19. Nota. SCR: Standart Competition Ranking, representación total de documentos.

\begin{tabular}{ccccc}
\hline SCR & Autores & $\begin{array}{c}\text { Documentos } \\
\text { publicados }\end{array}$ & Citaciones & $\begin{array}{c}\text { Fuerza de enlace } \\
\text { entre investigadores }\end{array}$ \\
\hline 1 & Kuldeep Dhama & 11 & 152 & 29 \\
2 & Ruchi Tiwari & 9 & 148 & 29 \\
3 & Shailesh Patel & 7 & 140 & 27 \\
4 & Ranjit Sah & 5 & 135 & 28 \\
5 & Yashpal Malik & 6 & 105 & 29 \\
6 & Kumar Seang & 6 & 125 & 20 \\
7 & Po-ren Hsueh & 6 & 2145 & 32 \\
8 & Seong-Jun Kim & 5 & 456 & 26 \\
9 & Changchuan Yin & 5 & 97 & 10 \\
10 & Yung Yuen & 5 & 17 & 3 \\
\hline
\end{tabular}


Rev. Cient. Cien. Nat. Ambien. 15(2):289-298 Diciembre 2021
Llerena • Sars-Cov-2 Un Análisis Bibliométrico en La Actualidad

Tabla 2. Top 10 de instituciones más productivas en la temática del covid-19. Nota. SCR: Standart Competition Ranking, representación total de documentos.

\begin{tabular}{|c|c|c|c|c|}
\hline SCR & Organizaciones & $\begin{array}{l}\text { Documentos } \\
\text { publicados }\end{array}$ & Citaciones & $\begin{array}{l}\text { Fuerza de enlace } \\
\text { entre instituciones }\end{array}$ \\
\hline 1 & Chinese Academy of Sciences & 19 & 628 & 8 \\
\hline 2 & Huazhong University of Science and Technology & 34 & 526 & 6 \\
\hline 3 & Fudan University & 11 & 228 & 4 \\
\hline 4 & Zhejiang University & 13 & 192 & 2 \\
\hline 5 & Indian Veterinary Research Institute Izatnagar & 12 & 159 & 1 \\
\hline 6 & The University of Hong Kong & 12 & 322 & 5 \\
\hline 7 & Tabriz University of Medical Sciences & 9 & 57 & 3 \\
\hline 8 & Università degli Studi di Milano Statale & 14 & 93 & 3 \\
\hline 9 & Universidad de São Paulo & 9 & 88 & 4 \\
\hline 10 & Wuhan University & 10 & 106 & 2 \\
\hline
\end{tabular}

Tabla 3. Base de datos (Web of Science, Scopus y PubMed). Nota. SCR: Standart Competition Ranking, representación total de documentos.

\begin{tabular}{|c|c|c|c|c|}
\hline Años & Web of Science & Scopus & PubMed & $\begin{array}{c}\text { Documentos } \\
\text { publicados }\end{array}$ \\
\hline 2021 & 46.418 & 21.894 & 15.856 & 84.168 \\
\hline 2020 & 43.803 & 17.431 & 12.701 & 73.935 \\
\hline 2019 & 16 & 10 & 7 & 23 \\
\hline 2018 & 1 & 1 & 1 & 3 \\
\hline 2017 & 1 & 0 & 0 & 1 \\
\hline
\end{tabular}

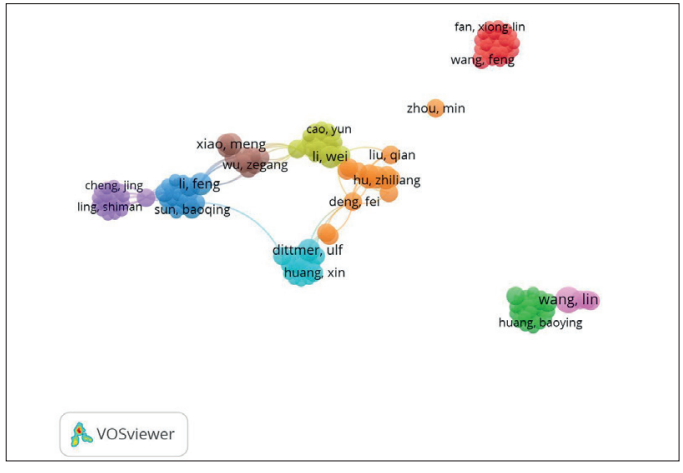

Figura 3. Principales autores en investigaciones sobre la covid-19.

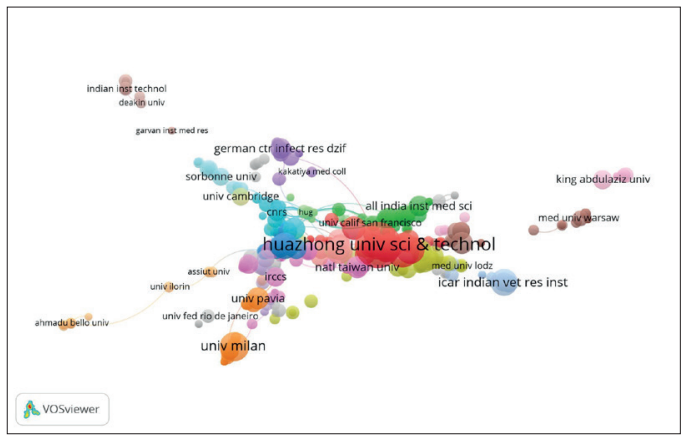

Figura 4. Principales organizaciones en áreas de covid-19. 
Rev. Cient. Cien. Nat. Ambien. 15(2):289-298

Diciembre 2021

Tabla 4. Top 10 de países que investigan sobre la covid-19. Nota. SCR: Standart Competition Ranking, representación total de documentos.

\begin{tabular}{ccccc}
\hline SCR & Países & $\begin{array}{c}\text { Documentos } \\
\text { publicados }\end{array}$ & Citaciones & $\begin{array}{c}\text { Fuerza de enlace } \\
\text { entre países }\end{array}$ \\
\hline 1 & Estados Unidos & 211 & 3522 & 74.00 \\
2 & China & 201 & 5714 & 39.00 \\
3 & India & 88 & 699 & 29.00 \\
4 & Alemania & 68 & 925 & 23.00 \\
5 & Inglaterra & 52 & 796 & 22.00 \\
6 & Italia & 105 & 1307 & 22.00 \\
7 & Irán & 35 & 390 & 17.00 \\
8 & España & 47 & 646 & 12.00 \\
9 & Brasil & 40 & 335 & 11.00 \\
\hline 10 & Turquía & 40 & 432 & 10.00 \\
\hline
\end{tabular}

Tabla 5. Top 10 de idiomas de publicaciones sobre la covid-19. Nota. SCR: Standart Competition Ranking, representación total de documentos.

\begin{tabular}{ccc}
\hline SCR & Idiomas & Documentos publicados \\
\hline 1 & Inglés & 85.882 \\
2 & Español & 1.775 \\
3 & Alemán & 693 \\
4 & Portugués & 552 \\
5 & Francés & 306 \\
6 & Ruso & 303 \\
7 & Italiano & 260 \\
8 & Turco & 208 \\
9 & Chino & 63 \\
10 & Húngaro & 55 \\
\hline
\end{tabular}


Rev. Cient. Cien. Nat. Ambien. 15(2):289-298 Diciembre 2021
Llerena • Sars-Cov-2 Un Análisis Bibliométrico en La Actualidad

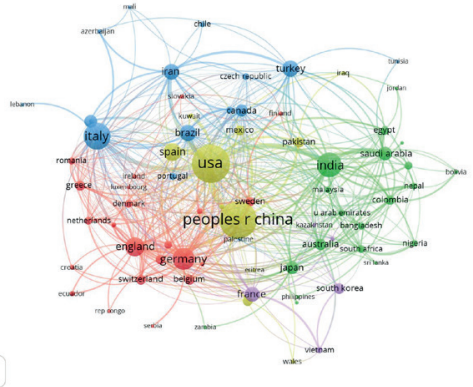

Figura 5. Principales países en investigaciones sobre el coronavirus.

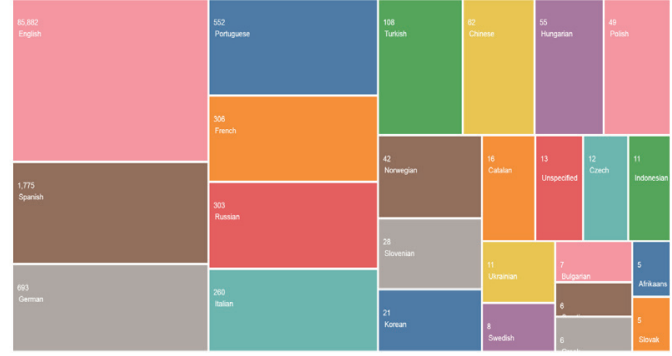

Figura 6. Otros idiomas de publicaciones científicas sobre la covid-19.

Tabla 6. Top 10 de las áreas de investigaciones sobre la covid-19. Nota. SCR: Standart Competition Ranking, representación total de documentos.

\begin{tabular}{ccc}
\hline SCR & Áreas & Documentos publicados \\
\hline 1 & Medicina interna general & 8.966 \\
2 & Salud ocupacional ambiental pública & 8.819 \\
3 & Ciencia y tecnología & 5.919 \\
4 & Ecología en ciencias ambientales & 4.987 \\
5 & Enfermedades infecciosas & 4.385 \\
6 & Negocios económicos & 4.014 \\
7 & Psicología & 3.788 \\
8 & Servicios de ciencias de la salud & 3.483 \\
9 & Inmunología & 3.042 \\
10 & Farmacología & 2.814 \\
\hline
\end{tabular}

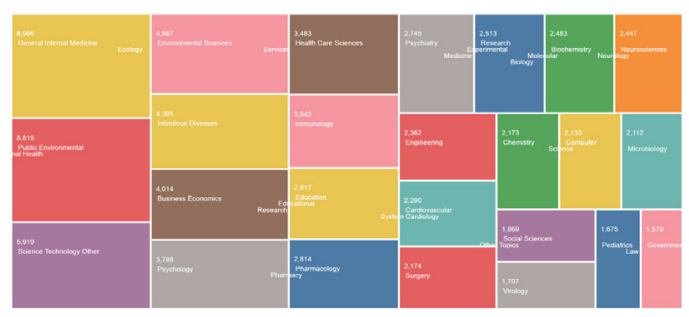

Figura 7. Otras áreas relacionadas a la medicina y al covid-19.

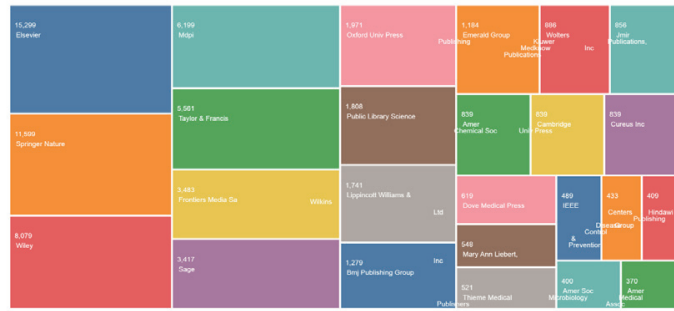

Figura 8. Otros editores que hablan acerca del tema del covid-19. 
Tabla 7. Top 10 de los editores científicos sobre la covid-19. Nota. SCR: Standart Competition Ranking, representación total de documentos.

\begin{tabular}{ccc}
\hline SCR & Editores & Documentos publicados \\
\hline 1 & Elsevier & 15.299 \\
2 & Springer Nature & 11.599 \\
3 & Wiley & 8.079 \\
4 & Multidisciplinary Digital Publishing Institute & 6.199 \\
5 & Taylor y Francis & 5.561 \\
6 & Frontiers Media & 3.483 \\
7 & Sage & 3.417 \\
8 & Oxford Univ Press & 1.971 \\
9 & Public Library Science & 1.808 \\
10 & Lippincott Williams y Wilkins & 1.741 \\
\hline
\end{tabular}

Tabla 8. Top 10 de palabras claves sobre la covid-19. Nota. SCR: Standart Competition Ranking, representación total de documentos.

\begin{tabular}{cccc}
\hline SCR & Palabras claves & Ocurrencias & $\begin{array}{c}\text { Fuerza de enlaces } \\
\text { entre palabras claves }\end{array}$ \\
\hline 1 & Covid-19 & 919 & 1.712 \\
2 & Sars-CoV-2 & 908 & 1.708 \\
3 & Coronavirus & 301 & 860 \\
4 & Neumonía & 111 & 377 \\
5 & Infección & 106 & 328 \\
6 & Mortalidad & 92 & 300 \\
7 & Sars & 81 & 296 \\
8 & $2019-n C o V$ & 80 & 268 \\
9 & Transmisión & 77 & 247 \\
10 & Virus & 63 & 222 \\
\hline
\end{tabular}

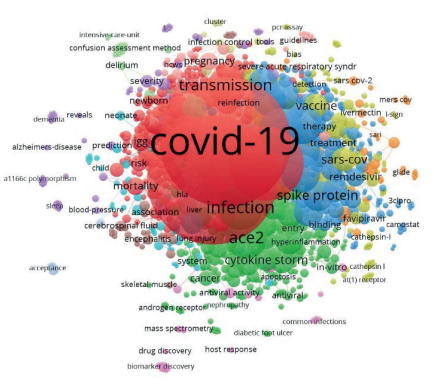

Figura 9. Otros ejes temáticos vinculados a la covid-19. covid-19. Nota. SCR: Standart Competition Ranking, representación total de documentos.

\section{Conclusiones}

Las primordiales conclusiones del presente trabajo se desglosan en los siguientes apartados: 1) Kuldeep Dhama es el autor más productivo en temas del covid-19; 2) la universidad que aporta con más investigaciones es Chinese Academy of Sciences; 3) el año de publicación que se registró más relevancia en investigaciones de contextos médicos (Sars-CoV-2) fue el 2021 ; 4) el país que mayor influencia tuvo 
en investigaciones sobre el coronavirus es Estados Unidos; 5) el idioma que prevaleció en las distintas publicaciones es el inglés; 6) la principal área de investigación referente al campo medico es la medicina interna general; 7) el editor más significativo es Elsevier con más de 15.299 documentos publicados y 8 ) la palabra clave más utilizada para la búsqueda de información referente al tema de estudio es "covid-19" (Backer et al., 2020; Liu., 2020; Masters, 2006; Ryu y Chun, 2020). Finalmente, es importante destacar que la rápida expansión del covid-19 y sus diferentes cepas generarán futuras investigaciones centrándose en las eficiencias de las vacunas más nombradas (Pfizer, AstraZeneca, Moderna, Sinovac, Sputnik, etc...) donde se espera que exista mayor información estadística y producción científica para poder realizar nuevos estudios bibliométricos en otras databases como ScienceDirect, Crossref, Springer y Jstor (Chang, 2020; Chen et al., 2020; Elfiky, 2020; Jaffar, 2020; Jon, 2020; Robson, 2020; Zunyou y McGoogan, 2020).

\section{Referencias}

Backer, J., Klinkenberg, D., y Wallinga, J. 2020. Incubation period of 2019 novel coronavirus (2019-nCoV) infections among travelers from Wuhan, China. Euro Surveil, 5, 8996. doi: DOI: 10.2807/1560-7917.ES.2020.25.5.2000062

Bwire, G., y Paulo, L. 2020. Coronavirus disease-2019: is fever an adequate screening for the returning travelers? Tropical Medicine and Health, 48(14), 45-55. doi:https:// doi.org/10.1186/s41182-020-00201-2

Chahrour, M., Assi, S., Bejjani, M., Nasrallah, A., Salhab, H., y Farares, M. 2020. A bibliometric analysis of Covid-19 research activity: A call for increased output. Cureus, 12(3), 456-465. doi:10.7759/cureus.7357

Chang, L. 2020. Pay attention to situation of SARS-CoV-2 and TCM advantages in treatment of novel coronavirus infection. Chinese Herbal Medicines, 12(2), 97-103. doi:https://doi.org/10.1016/j.chmed.2020.03.004

Chang, Y. B., y Gurbaxani, V. 2012. Information Technology Outsourcing, Knowledge Transfer, and Firm Productivity. MIS Quarterly, 36(4), 1043-1063.

Chen, J., Wenxiong, X., Ziying, L., Jing, L., y Liang, P. 2020. Recurrence of positive SARS-CoV-2 RNA in COVID-19: A case report. International Journal of Infections Diseases, 93, 67-75. doi:https://doi.org/10.1016/j.ijid.2020.03.003

Chen, J.-S., y Tsou, H.-T. 2012. Performance Effects of IT Capability, Service Process Innovation, and the Mediating Role of Customer Service. Journal of Engineering and Technology Management, 29(1), 71-94.

Cheng, C., Ping, T., Chien, W., Jen, H., y Ren, P. 2020. Severe acute respiratory syndrome coronavirus 2 (SARS-CoV-2) and coronavirus disease-2019 (COVID-19): The epidemic and the challenges. International Journal of Antimicrobial Agents, 55, 23-31. doi:https://doi.org/10.1016/j. ijantimicag.2020.105924

Dumas, M., Garenne, A., Boraud, T., y Gonon, F. 2020. Does newspapers coverage influence the citations count of scientific publications? An analysis of biomedical studies. Scientometrics, 413-427. doi:https://doi.org/10.1007/ s11192-020-03380-1

Elfiky, A. 2020. Ribavirin, Remdesivir, Sofosbuvir, Galidesivir, and Tenofovir against SARS-CoV-2 RNA dependent RNA polymerase (RdRp): A molecular docking study. Life Sciences, 253, 80-86. doi:https://doi.org/10.1016/j. Ifs. 2020.117592

Giwan, S., Geonhee, L., Mi, J., Seung-Hwa, B., Minsuk, C.,
Keun, B., Chang-Seop, L., Sangmi, J., Daeui, P., Hong, G. K., Seong-Jun, K., Jeong-O, L., Bum T. K., Edmond, C. P., Seung, K. 2020. Rapid Detection of COVID-19 Causative Virus (SARS-CoV-2) in Human Nasopharyngeal Swab Specimens Using Field-Effect Transistor-Based Biosensor. Acs Nano, 14, 5135-5142. doi:https://dx.doi.org/10.1021/ acsnano.0c02823

Jaffar, A. 2020. Asymptomatic coronavirus infection: MERS-CoV and SARS-CoV-2 (COVID-19). Travel Medicine and Infectious Disease, 35, 34-39. doi:https://doi. org/10.1016/j.tmaid.2020.101608

Jianju, G., Zhenxue, T., y Xu, Y. 2020. Chloroquine phosphate has shown apparent efficacy in treatment of COVID-19 associated pneumonia in clinical studies. Biosci Trends, 14(1), 72-76. doi:https://pubmed.ncbi.nlm.nih. gov/32074550/\#: : :text=1)\%3A72-73.-,doi\%3A\%2010.5582/ bst.2020.01047,-

Jon, D. 2020. New SARS-like virus in China triggers alarm. Science, 367(6475), 234-235. doi:DOI: 10.1126/ science.367.6475.234

Kanne, J. 2020. Chest CT findings in 2019 novel coronavirus (2019-nCoV) infections from Wuhan, China: key points for the radiologist. Radiology, 291(1), 16-20. doi: DOI: 10.1148/radiol.2020200241

Lai, C., Shih, T., Ko, W., Tang, J., y Hsueb, P. 2020. Severe acute respiratory syndrome acute respiratory syndrome and coronavirus disease-2019 COVID-19): the epidemic and the challenges. International Journal of Antimicrobial Agents, 55(3), 45-50. doi:https://doi.org/10.1016/j. ijantimicag.2020.105924

Lawani, S. 1981. Bibliometrics: Its Theoretical Foundations. Journal Libri, 31(1), 234-245. doi:https://doi.org/10.1515/ libr.1981.31.1.294

Liu, H., Ke, W., Wei, K. K., y Hua, Z. 2013. The Impact of IT Capabilities on Firm Performance: The Mediating Roles of Absorptive Capacity and Supply Chain Agility. Decision Support Systems, 54(3), 1452-1462.

Liu, K., Yuan, Y., Liu, W., Wang, M., y Cheng, L. H. 2020. Clinical characteristics of novel coronavirus cases in tertiary hospitals in Hubei Province. Chinese Medical Journal, 133(9), 1025-1031. doi:doi: 10.1097/ CM9.0000000000000744

Llerena, M., y Arévalo, E. 2020. Análisis bibliométrico de la interacción profesor - alumno a través de las plataformas virtuales. Espíritu Emprendedor TES, 4(4), 42-65.

Lu, H. 2020. Drug treatment options for the 2019-new coronavirus (2019-nCoV). Biosci Trends, 14(1), 69-71. doi:10.5582/bst.2020.01020

Masters, P. 2006. The molecular biology of coronaviruses. Adv Virus Res, 66, 193-292. doi:DOI: 10.1016/\$00653527(06)66005-3

Matias-Matias-Guiu, J. 2020. The role of scientific journal editors during the COVID-19 pandemic. Neurologia, 35(4), 223-225. doi:https://dx.doi.org/10.1016/j. nrleng.2020.05.011

O'Brien, N., Barboza, M., Ventura, J., Caycho, R., Sandoval, S., López, W., y Salas, G. 2020. Nuevo coronavirus (COVID-19) Un análisis bibliométrico. Revista Chilena de Anestesia, 49(1), 408-415. doi:10.25237/revchilanestv49n03.020

Pascual, C., y Torrell, v. 2020. Bibliometric analysis of Latin American and Caribbean scientific production about COVID-19 in pubmed. Revista Cubana de Información en Ciencias de La Salud, 31(3), 1-21. doi:https://doi. org/10.36512/rcics.v31i3.1600

Phat, T. 2020. Genetic diversity and evolution of SARSCoV-2. Infect. Genet. Evol., 81. doi:10.1016 j.meegid.2020.104260.

Robson, B. 2020. Computers and viral diseases. Preliminary bioinformatics studies on the design of a synthetic vaccine and a preventative peptidomimetic antagonist against the SARS-CoV-2 (2019-nCoV, COVID-19) coronavirus. Computers in Biology and Medicine, 119, 78-82. doi:https://doi. 
org/10.1016/j.compbiomed.2020.103670

Ryu, S., y Chun, B. 2020. Epidemiological characteristics of 2019 novel coronavirus: an interim review. Epidemiol Health, 42, 46-51. doi:DOI: https://doi.org/10.4178/epih. e2020006

Shuai, X., Yun, Z., Meiquin, L., Qiaoshuai, L., Wei, X., Yanling, W., Tianlei, Y., Shuwen, L., Zhengli, Z., Shubo, J., Lu, L. 2020. Fusion mechanism of 2019-nCoV and fusion inhibitors targeting HR1 domain in spike protein. Cellular \& Molecular Immunology, 17, 765-767. doi:https://doi. org/10.1038/s41423-020-0374-2

van Eck, N.J. y Waltman, L. 2010. Software survey: VOSviewer, a computer program for bibliometric mapping. Scientometrics, 84, 523-538. doi:https://link.springer. com/article/10.1007/s11192-009-0146-3

Vásquez, K., Roque, J., Angulo, Y., y Ninatanta, J. 2021. Análisis bibliométrico de la producción científica Peruana sobre la covid-19. Revista Peruana de Medicina de Experimental y Salud Pública, 38(2), 34-45. doi:https:// doi.org/10.17843/

Wang, D. H., y Huynh, Q. L. 2013. Mediating Role of Knowledge Management in Effect of Management Accounting Practices on Firm Performance. Journal of Knowledge Management, Economics and Information Technology, 3(3), 1-25.

Wang, M., y Liao, Z. 2020. SARS-CoV-2 and COVID-19: How much do we know? Acta virologica, 64, 288-296. doi:doi: 10.4149/av_2020_301

Wu, F., Wang, A., Liu, M., Wang, Q., Chen, J., Xia, S., y Huang, J. (s.f.). 2020. Neutralizing Antibody Responses to SARS-CoV-2 in a COVID-19 Recovered Patient Cohort and Their Implications. medRxiv. doi:https://doi. org/10.1101/2020.03.30.20047365

Zhe, X., Lei, S., y Yijin, W. 2020. Pathological findings of COVID-19 associated with acute respiratory distress syndrome. The Lancet Respiratory Medicine, 8(4), 420422. doi:https: //doi.org/10.1016/s2213-2600(20)30076-X

Zhou, P., P., Yang, X., Wang, X., Hu, B., Zhang, L., Zhang, W., Si, H., Zhu, Y., Li, B., Huang, C., Chen, H, Chen, J., Luo, Y., Guo, H., Jiang, R., Liu, M., Chen, Y., Shen, X., Wang, X., Zheng, X., Zhao, K., Chen, Q., Deng, F., Liu, L., Yan, N., Zhan, F., Wang, Y., Xiao, G. y Shi, Z. 2020. A pneumonia outbreak associated with a new coronavirus of probable bat origin. Nature, 579, 270-273. doi:https://doi. org/10.1038/s41586-020-2012-7

Zunyou, W., y McGoogan, J. 2020. Characteristics of and Important Lessons From the Coronavirus Disease 2019 (COVID-19) Outbreak in China. Jama, 323(13), 1239-1242. doi:doi:10.1001/jama.2020.2648 